\title{
Waves of magnetic moment and generation of waves by neutron beam in quantum magnetized plasma
}

\author{
P. A. Andreev \\ Department of General Physics, Faculty of Physics, \\ Moscow State University, Moscow, Russian Federation. \\ L. S. Kuzmenkovi \\ Department of Theoretical Physics, Physics Faculty, \\ Moscow State University, Moscow, Russian Federation.
}

(Dated: July 25, 2018)

\begin{abstract}
This paper is devoted to studying of dispersion of waves in the magnetized plasma with the spin and exploring of new methods of the generation wave in the plasma. We consider the dispersion of waves, existed in the plasma in consequence of dynamic of the magnetic moments. It is shown there are nine new waves in the magnetized plasma because of the magnetic moments dynamic. We show there are instabilities at propagation of the neutron beam through the plasma. Increments of instabilities caused by neutron beam are calculated. For studying of this effects we generalize and use the method of the many-particle quantum hydrodynamics. Described processes can play important role at calculation of the stability and the safeness of the nuclear reactors and the studying of the processes in the atmosphere of the neutron stars.
\end{abstract}

\section{INTRODUCTION}

In plasma physics the effect of generation of waves by means of an electron beam is well known [1]- [3] and some aspects of this problem have been studied [4]- [11]. The self-consistent electromagnetic interaction of the electrons of the beam with the electrons and ions of the plasma plays a crucial role. The increasing of an amplitude of collective oscillation arises. At these processes the generation of waves takes place in both cases at the propagation of the beam through the plasma and at the propagation of the beam along a space-limited medium due to the long-range electromagnetic interaction.

Plasma is the isotropic medium. At presence of the uniform external magnetic field the preferential direction is arisen - the direction of the external magnetic field. In this case the properties of the plasma change essentially. Dispersion dependence of waves changes. Besides new types of waves emerge. The dispersion properties of these waves essentially depend on the angle between the direction of the wave propagation and the direction of the external magnetic field.

In our work we consider the plasma in an external uniform magnetic field. We regard the motion of both the electrons and the ions. We pay attention to the spin of particles, more precisely their magnetic moments. The dynamic of magnetic moments leads to change in the dispersion dependence of excitations, which exist in the plasma at the absence of magnetic moments. It also leads to arising of new waves. The two waves arise at propagation along the external magnetic field and the four waves emerge at propagation perpendicular to the external field.

Special attention, we give to the process of the interaction of a neutron beam with the plasma. Neutrons have magnetic moment. Consequently, they can interact with the spins and the currents in the plasma by means of the collective magnetic field. In that process, the beam of neutrons transfers energy to the plasma and generates waves. We focus our attention on case when the neutron beams moving in the direction of the external magnetic field. We also consider an opportunity of generation of the waves which propagate along the external magnetic field.

In last years there have been a lot of papers devoted to influence of spin on dynamic of the plasma. Brief review of this studies is presented in Ref.s [12-14]. Recently, the quantum kinetic studying of the waves in the plasma was made [15]. Contribution of the semi-relativistic effects was studied there. Another examples of derivation of the quantum kinetic equation are considered in Ref.s [16, 17].

Moreover, we make further development of the method of investigation of similar problem, which is called the many-particle quantum hydrodynamics.

The Vlasov kinetic equation or the corresponding hydrodynamic equations for system of charged particles is used in the classic physics at investigation of plasma properties.

Consistent investigation of dynamics of magnetic moment or spin of particles expects of the quantummechanical description. Consequently, we derive equations of the collective quantum motion. We obtain them from the many-particle Schrodinger equation. They arise in the form similar to the hydrodynamics equations. Therefore, the developing method is called quantum hydrodynamics (QHD). For the first time, for many-body system, this method was proposed in paper [18]. Its generalization for the system of spinning particles was made in Ref. [19]. Another method of derivation of the QHD equations is proposed in paper [20]. Relativistic generalization of this approximation was made in Ref. [21]. We study the contribution of the spin-current and the spin-orbit interactions. The spin-current interaction is the interaction between the 
moving charge and the motionless spin, more precisely magnetic moments. The spin-orbit interactions is the interaction between the motionless charged particles and the moving spin. For the system of two particles one of the described interactions could be exclude. We can make it by using appropriate frame. But for many-body system we need to consider both of them. The system of the quantum hydrodynamic equations in the self-consistent field approximation looks like the same obtained from the Schrodinger equation for one particles in external field. The many-body QHD contains a quantum correlation. We do not consider correlation in this paper. Good example of calculation of the correlations is presented in Ref. [22], where evolution of neutral quantum particles is studied. In this case there is no self-consistent field and interaction determined by the correlations only.

We make further development of fundamental results obtained in Ref.s [19] and [23]. We generalize method of the QHD [19] including the spin-current and the spin-orbit interactions along with the spin-spin and the Coulomb interactions between particles. Effect of increasing of instabilities at the propagation of the neutron beam through the magnetized plasma was predicted in [23]. Magnetic moment of the neutron plays crucial role in this effect. This effect is consequence of the spin-spin and the spin-current interaction between the spins of the beam and the spin and current of the plasma. Here we study the influence of the spin-orbit interaction on the described effect. This effect can be called the effect of the resonant interaction of the neutron beam with the plasma. In the part of the paper, where we consider the propagation of the beam through the medium, we bound oneself by the case there both the beam and waves propagate along the external magnetic field.

We are interested in instabilities which arise at interaction of the beam with both the well-known magnetoplasma and the recently predicted waves [24], [25]. In this work we do not present the amendments, caused by the spin, in the dispersion of the well-known waves. Readers who are interested in this subject see Ref.s [12] and [24].

This paper is organized as follows. In Sec. II we present the system of the quantum hydrodynamic equations. In Sec. III we describe the method of solving of the equations for the case of linear excitations. In Sec. IV we study dispersion of new type of waves in the plasma caused by dynamic of magnetic moments. We examine the waves propagated along the external magnetic field. We research propagation of the neutron beam through the plasma. We consider interaction of the beam with both the well-known plasma waves and the new waves caused by spin dynamics. We show existence of instabilities in this case and calculate the increments of the instabilities. In Sec. V we consider waves propagated perpendicular to the external magnetic field. We interested in dispersion of waves caused by spin dynamics. In Sec. VI the dispersion of the spin waves is obtained. We make special attention for the spin waves dis- persion in the whole $\mathbf{k}$ space. In Sec. VII we present the brief summary of our results.

\section{THE MODEL}

The equations of quantum hydrodynamic are derived from the non-stationary Schrodinger equation for system of $\mathrm{N}$ particles:

$$
\begin{gathered}
\imath \hbar \partial_{t} \psi(R, t)=\left(\sum_{n}\left(\frac{1}{2 m_{n}} \mathbf{D}_{n}^{2}+e_{n} \varphi_{n, e x t}\right)\right. \\
\left.+\sum_{n, k \neq k} \frac{1}{2} e_{n} e_{k} G_{n k}\right) \psi(R, t) .
\end{gathered}
$$

The following designations are used in the equation (1): $D_{n}^{\alpha}=-\imath \hbar \partial_{n}^{\alpha}-e_{n} A_{n, \text { ext }}^{\alpha} / c, \varphi_{n, \text { ext }}, A_{n, \text { ext }}^{\alpha}$ - the potentials of the external electromagnetic field,

$$
\partial_{n}^{\alpha}=\nabla_{n}^{\alpha}=\frac{\partial}{\partial x_{n, \alpha}},
$$

and $G_{n k}=1 / r_{n k}$, - is the Green functions of the Coulomb interaction, $\psi(R, t)$-is psi function of $\mathrm{N}$ particle system, $R=\left(\mathbf{r}_{1}, \ldots, \mathbf{r}_{N}\right), e_{n}, m_{n}$-are the charge and the mass of particle, $\hbar$-is the Planck constant and $c$ is the speed of light.

We consider the interaction between particles and action of the external electromagnetic field on them.

The method of the QHD is based on the Schrodinger equation, which is the non-relativistic. But the Schrodinger equation allows to account the semi-relativistic amendments, accurate to $v^{2} / c^{2}$. In this work we interested in the spin-spin, the spin-current and the spin-orbit interactions along with the Coulomb interaction. In this section we present the Hamiltonian included the Coulomb interaction only. We made it for simplicity. Resulted equations, see below in this section, contain all described interactions. Whole Hamiltonian is presented in the Appendix.

Here we briefly present the basic steps of derivation of the QHD equation. More detailed description of derivation of the many-particle QHD equations can be find in [19].

The first step in derivation of equations of the QHD is a definition of concentration of particles. We determine the concentration of particles as quantum-mechanical average of the operator of concentration:

$$
\hat{n}=\sum_{n} \delta\left(\mathbf{r}-\mathbf{r}_{n}\right) .
$$

This function is the microscopic function of concentration in classic physics, $\delta(\mathbf{r})$-is the Dirac's $\delta$-function.

In that way, the concentration has form:

$$
n(\mathbf{r}, t)=\int d R \sum_{n} \delta\left(\mathbf{r}-\mathbf{r}_{n}\right) \psi^{*}(R, t) \psi(R, t),
$$

where $d R=\prod_{n=1}^{N} d \mathbf{r}_{n}$. 
Differentiating the concentration with respect to time, using the Schrodinger equation and evident form of the Hamiltonian, we obtain the continuity equation:

$$
\partial_{t} n(\mathbf{r}, t)+\nabla \mathbf{j}(\mathbf{r}, t)=0 .
$$

In that equation a function of current $\mathbf{j}(\mathbf{r}, t)=$ $n(\mathbf{r}, t) \mathbf{v}(\mathbf{r}, t)$ is arisen, where $\mathbf{v}(\mathbf{r}, t)$ - is the velocity field.

Differentiating the function of current with respect to time, we obtain the momentum balance equations, this equation is an analog of the Euler equation:

$$
\begin{gathered}
m n(\mathbf{r}, t)\left(\partial_{t}+v^{\beta}(\mathbf{r}, t) \nabla^{\beta}\right) v^{\alpha}(\mathbf{r}, t)+\partial_{\beta} p^{\alpha \beta}(\mathbf{r}, t) \\
-\frac{\hbar^{2}}{4 m} \partial^{\alpha} \triangle n(\mathbf{r}, t)+\frac{\hbar^{2}}{4 m} \partial^{\beta}\left(\frac{\partial^{\alpha} n(\mathbf{r}, t) \cdot \partial^{\beta} n(\mathbf{r}, t)}{n(\mathbf{r}, t)}\right) \\
=e n(\mathbf{r}, t) E^{\alpha}(\mathbf{r}, t)+\frac{e}{c} \varepsilon^{\alpha \beta \gamma} n(\mathbf{r}, t) v^{\beta}(\mathbf{r}, t) B^{\gamma}(\mathbf{r}, t) \\
+M^{\beta}(\mathbf{r}, t) \nabla^{\alpha} B^{\beta}(\mathbf{r}, t)+F_{s-o}^{\alpha}(\mathbf{r}, t),
\end{gathered}
$$

where $\mathbf{E}$ and $\mathbf{B}$ are the electric and magnetic fields, $\mathbf{M}$ is the density of magnetic moments, $\varepsilon^{\alpha \beta \gamma}$ - is the antisymmetric symbol (the Levi-Civita symbol), $p^{\alpha \beta}$ is the kinetic pressure tensor.

Terms which proportional to $\hbar^{2}$ is the quantum Bohm potential, they appear as a result of using of quantum mechanics. In right-hand side of equation (4) a force field locates. The force field consists of the Lorenz force, force of interaction of spins with magnetic field and spin-orbit interaction. Form of force which act on the magnetic moments is differ from analogous in the mechanics, which has the form of $\mathbf{F}_{m e c}=(\mathbf{M} \nabla) \mathbf{B}$.

The force field for the spin-orbit interaction $\mathbf{F}_{s-o}$ arises in the form:

$$
\begin{aligned}
F_{s-o}^{\alpha}(\mathbf{r}, t)= & \frac{1}{c} \frac{2 \gamma}{\hbar} \varepsilon^{\alpha \beta \mu} \varepsilon^{\beta \gamma \delta} B^{\gamma}(\mathbf{r}, t) M^{\delta}(\mathbf{r}, t) E^{\mu}(\mathbf{r}, t) \\
& -\frac{1}{c} \varepsilon^{\alpha \beta \gamma} M^{\beta}(\mathbf{r}, t) \partial_{t} E^{\gamma}(\mathbf{r}, t) \\
& -\frac{1}{c} \varepsilon^{\alpha \beta \gamma} \partial^{\delta} E^{\gamma}(\mathbf{r}, t) J_{M}^{\beta \delta}(\mathbf{r}, t) \\
& -\frac{1}{c} \varepsilon^{\beta \gamma \mu} J_{M}^{\beta \gamma}(\mathbf{r}, t) \partial^{\alpha} E^{\mu}(\mathbf{r}, t)
\end{aligned}
$$

where $J_{M}^{\alpha \beta}(\mathbf{r}, t)$ - is the tensor of current of magnetic moment.

In this paper we obtain contribution of the spin-orbit interaction in the Euler equation and equation of balance of magnetic moment.

The equation of evolution of the magnetic moment is:

$$
\begin{aligned}
& \partial_{t} M^{\alpha}(\mathbf{r}, t)+\nabla^{\beta} J_{M}^{\alpha \beta}(\mathbf{r}, t) \\
= & \frac{2 \gamma}{\hbar} \varepsilon^{\alpha \beta \gamma}\left(M^{\beta}(\mathbf{r}, t) B^{\beta}(\mathbf{r}, t)\right.
\end{aligned}
$$

$$
\left.+\frac{1}{c} \varepsilon^{\beta \mu \nu} J_{M}^{\gamma \nu}(\mathbf{r}, t) E^{\mu}(\mathbf{r}, t)\right) .
$$

This equation is analog of the Bloch equation. Last term is the contribution of the spin-orbit interaction.

This equations take place for each species of particles. They connected by means of the Maxwell's equations:

$$
\begin{gathered}
\nabla \mathbf{B}(\mathbf{r}, t)=0, \quad \nabla \mathbf{E}(\mathbf{r}, t)=4 \pi \sum_{a} e_{a} n_{a}(\mathbf{r}, t), \\
\nabla \times \mathbf{E}(\mathbf{r}, t)=-\frac{1}{c} \partial_{t} \mathbf{B}(\mathbf{r}, t) \\
\nabla \times \mathbf{B}(\mathbf{r}, t)=\frac{1}{c} \partial_{t} \mathbf{E}(\mathbf{r}, t) \\
+\frac{4 \pi}{c} \sum_{a} e_{a} n_{a}(\mathbf{r}, t) \mathbf{v}_{a}(\mathbf{r}, t)+4 \pi \sum_{a} \nabla \times \mathbf{M}_{a}(\mathbf{r}, t),
\end{gathered}
$$

where subindex "a" describe the kind of particle: electrons, ions and neutrons.

The tensor of the magnetic moments flow $J_{M}^{\alpha \beta}$ may be approximately presented in the form $J_{M}^{\alpha \beta}=M^{\alpha} v^{\beta}$. This is true if we do not consider the thermal motion of magnetic moments.

\section{DISPERSION EQUATION}

We consider the small perturbation of equilibrium state like

$$
\begin{gathered}
n_{a}=n_{0 a}+\delta n_{a}, \mathbf{E}=0+\mathbf{E}, \\
\mathbf{B}=B_{0} \mathbf{e}_{z}+\delta \mathbf{B}, \mathbf{v}_{a}=0+\mathbf{v}_{a}, \\
\mu_{a}^{\alpha}=\mu_{0 a}^{\alpha}+\delta \mu_{a}^{\alpha}, M_{0 a}^{\alpha}=n_{0 a} \mu_{0 a}^{\alpha}=\chi_{a} B_{0}^{\alpha}, \\
n_{0 e}=n_{0 i}, p_{a}^{\alpha \beta}=p_{a} \delta^{\alpha \beta}, \delta p_{a}=m_{a} v_{s a}^{2} \delta n_{a},
\end{gathered}
$$

here $\delta^{\alpha \beta}$ - is the Kronecker symbol, $\chi_{a}=\kappa_{a} / \nu_{a}$ is the ratio between the equilibrium magnetic susceptibility $\kappa_{a}$ and magnetic permeability $\nu_{a}=1+4 \pi \kappa_{a}$. In the case there $\kappa_{a} \ll 1$ we have $\chi_{a} \simeq \kappa_{a}$. In equations $(8), v_{s a}^{2}$ is the thermal velocity, for the case of degenerate electrons $v_{s a}^{2}$ is the Fermi velocity. Substituting these relations into the system of equations (3), (4), (6) and (7) and neglecting by nonlinear terms, we obtain a system of linear homogeneous equations in partial derivatives with constant coefficients. Passing to the following representation for small perturbations $\delta f$

$$
\delta f=f(\omega, \mathbf{k}) \exp (-\imath \omega+\imath \mathbf{k r})
$$

yields a homogeneous system of algebraic equations. The electric field strength is assumed to have a nonzero value. Expressing all the quantities entering the system of equations in terms of the electric field, we come to the equation

$$
\Lambda_{\alpha \beta}(\omega, \mathbf{k}) E_{\beta}(\omega, \mathbf{k})=0,
$$

Since the amplitude of the electric field is not equal to zero we have the dispersion equation

$$
\operatorname{Det} \widehat{\Lambda}(\omega, \mathbf{k})=0 \text {. }
$$

We do not present the explicit form of $\Lambda_{\alpha \beta}(\omega, \mathbf{k})$ because of its largeness. 


\section{THE WAVES PROPAGATED PARALLEL TO MAGNETIC FIELD}

In the absence of the neutron beam we have the magnetized plasma of particles with magnetic moment. Dispersion equation have two new solutions:

$$
\omega=\left|\Omega_{e}\right|\left(1+\frac{8 \pi k_{z}^{2} c^{2} \chi_{e}}{\omega_{L e}^{2}+2 k_{z}^{2} c^{2}-2 \Omega_{e}^{2}}\right),
$$

and

$$
\omega=\Omega_{i}\left(1-\frac{8 \pi k_{z}^{2} c^{2} \chi_{i}}{\omega_{L i}^{2}-2 k_{z}^{2} c^{2}+2 \Omega_{i}^{2}}\right),
$$

In this equation a follows notation is used $\omega_{L a}^{2}=$ $4 \pi e_{a}^{2} n_{0 a} / m_{a}$ - is the Langmuir frequency, $\Omega_{c}=$ $e_{c} B_{0} /\left(m_{c} c\right)$ that two parameters it is the cyclotron frequency which arise from the motion of the charge $e_{c}$ and the magnetic moment in the external magnetic field $B_{0}$ correspondingly, $\gamma_{a}$ is the gyromagnetic ratio, for example, for neutrons $\gamma_{b}=-1.91 \mu_{n u c}$, where $\mu_{n u c}$ is the nuclear magneton.

The frequency dependence of this waves is located around the electron or the ion cyclotron frequencies.

In the absence of the plasma we can obtain dispersion relations for the eigenwaves in the neutron beam. There are three solutions:

$$
\begin{gathered}
\omega^{2}=k^{2} c^{2} \mp 4 \pi \lambda k_{z} U_{z} W_{\gamma b}, \\
\omega=k_{z} U_{z} \pm \Omega_{\gamma b}-4 \pi \lambda W_{\gamma b}, \\
\omega=k_{z} U_{z} \mp \Omega_{\gamma b}+2 \pi \lambda\left(k_{z} U_{z} \mp \Omega_{\gamma b}\right) \frac{\Omega_{\gamma b} W_{\gamma b}}{k^{2} c^{2}},
\end{gathered}
$$

here and below $\lambda$ is equal to $1(\lambda=1)$ and indicate the contribution of the spin-orbit interaction, $W_{\gamma a}=\chi_{a} \Omega_{\gamma a}$, $\Omega_{\gamma a}=2 \gamma_{a} B_{0} / \hbar$, for charged particles we can use equality $\Omega_{\gamma a}=\Omega_{a}$.

Formulas (12) is a dispersion relation for the light which propagates through the neutron beam. Solution (14) arises in consequence of the spin-orbit interaction.

Now we consider the resonance interaction of mode of beam (13), (14) with waves in the plasma. We present results both for well-known waves and for the waves obtained in this paper.

First of all, we illustrate this effect on example of the fast magneto-sound wave. Let us consider the generation of the fast magneto-sound wave. The condition of the resonance interaction of the fast magneto-sound wave and beam mode with dispersion presented by formula (13) is $\omega_{0}=k_{z} U_{z}+\Omega_{\gamma b}$. Under this condition instabilities are arisen, increment of instabilities is presented by formula

$$
\delta \omega^{2}=-2 \pi \omega_{0}\left|W_{\gamma b}\right| \times
$$

$$
\times \frac{\lambda\left|\Omega_{\gamma b}\right|-2 \lambda k_{z} U_{z}+2\left(k_{z} c\right)^{2} / \omega_{0}}{2 \omega_{0}-\sum_{c} \omega_{L c}^{2} \Omega_{c} /\left(\omega_{0}+\Omega_{c}\right)^{2}}<0,
$$

where $\omega_{0}=\omega_{0}\left(k_{z}\right)$ - is the dispersion dependence of the fast magneto-sound wave in the absence of beam. For the fast magneto-sound wave where is $\omega_{0}(k) \subset\left(0,\left|\Omega_{e}\right|\right)$ and $2 \omega_{0}(k)-\sum_{c} \omega_{L c}^{2} \Omega_{c} /\left(\omega_{0}(k)+\Omega_{c}\right)^{2}>0$.

Resonance interaction of the wave (11) with beam mode (13) on condition $\Omega_{i}\left(1+\delta \Omega / \Omega_{i}\right)=k_{z} U_{z}+\Omega_{\gamma b}$ lead to the instability with

$$
\delta \omega^{2}=-\pi \times \frac{1}{1+\frac{3}{8} \frac{\omega_{L e}^{2}}{\left|\Omega_{e}\right| \Omega_{i}}} \times\left(2 \chi_{i}\left(k_{z} c\right)^{2}\right.
$$

$\left.+\left|W_{\gamma b}\right|\left(\lambda\left|\Omega_{\gamma b}\right|+2 k_{z} c\left(k_{z} c / \Omega_{i}-\lambda U_{z} / c\right)\right)\right)<0$.

Under condition $\omega_{A}(k)=k_{z} U_{z}-\Omega_{\gamma b}\left(\right.$ where $\omega_{A}(k)$ the dispersion dependence of the Alfven wave in absence of the beam, $\left.\omega_{A}(k) \in\left(0, \Omega_{i}\right)\right)$ there is the generation of the Alfven waves

$$
\begin{gathered}
\delta \omega^{2}=-2 \pi W_{\gamma b} \times \\
\times \frac{\lambda \omega_{A}(k) \Omega_{\gamma b}+2\left(k_{z} c\right)^{2}-2 \omega_{A} \lambda k_{z} U_{z}}{2 \omega_{A}(k)-\sum_{c} \omega_{L c}^{2} \Omega_{c} /\left(\omega_{A}(k)+\Omega_{c}\right)^{2}}<0 .
\end{gathered}
$$

at resonance with the beam mode (13).

In the case $\left|\Omega_{e}\right|\left(1+\delta \Omega /\left|\Omega_{e}\right|\right)=k_{z} U_{z}+\Omega_{\gamma b}$ there is the resonance interaction of the wave (10) with the beam mode (14) leading to the frequency shift

$$
\delta \omega= \pm \sqrt{\frac{32 \pi\left|\Omega_{e}\right|\left(\lambda\left|\Omega_{e} \Omega_{\gamma b} W_{\gamma b}\right|-2 k_{z}^{2} c^{2}\left|W_{\gamma e}\right|\right)}{\omega_{L e}^{2}-8 \Omega_{e}^{2}}} .
$$

For the dense plasma and conditions $k_{z}^{2} c^{2}>\left|\chi_{b} / \chi_{e}\right| \Omega_{\gamma b}^{2}$, $\left|\Omega_{\gamma e}\right|+\left|\Omega_{\gamma b}\right|=k_{z} U_{z}$ the solution (18) becomes imaginary, since condition for instabilities fulfills.

\section{PROPAGATION OF WAVES PERPENDICULAR TO MAGNETIC FIELD.}

In the case of the waves propagation perpendicular to the external magnetic field we consider only the propagation of waves and do not consider the interaction with the beam. We show the four new wave solutions arise in this case. Dispersion dependence of obtained waves has form:

$$
\omega=\left|\Omega_{a}\right|\left(1-\frac{2 \pi k^{2} c^{2} \chi_{a}}{\omega_{e}^{2}+k^{2} c^{2}-\Omega_{a}^{2}}\right) .
$$

In this paper we also report about another two solutions. In approximation of motionless ions one of presenting waves has follows dispersion relation

$$
\omega=\sqrt{\Omega_{e}^{2}+v_{q e}^{2} k_{\perp}^{2}}
$$




$$
+8 \pi^{2} \chi_{e}^{2} \frac{\Omega_{e}^{2}}{\sqrt{\Omega_{e}^{2}+v_{q e}^{2} k_{\perp}^{2}}} \times \frac{k_{\perp}^{4} c^{4}}{\omega_{e}^{2}\left(\omega_{e}^{2}+k_{\perp}^{2} c^{2}\right)},
$$

where

$$
v_{q s a}^{2}=v_{s a}^{2}+\frac{\hbar^{2} k^{2}}{4 m_{a}^{2}} .
$$

Formulas (19), (20) and all obtained in this paper solutions exist only under the conditions $\chi_{a} \neq 0$. They arise from dispersion equations containing new term proportional to $\chi_{a}$.

\section{SPIN WAVES}

Spin waves are the waves in which a perturbation of the electric field has no influence on process of the wave propagation. This waves propagate by means of the magnetic field.

Static magnetic field does not cause the electric field. When we consider a motion of charges or magnetic moments the magnetic field changes, it should lead to arises of the electric field according to the Maxwell's equations. At slow particles motion the magnetic field changes slowly. So, we can neglect the electric field which appears due to magnetic field changes. Charges also bring the electric field, but we suppose it give no contribution in the wave propagation. As a result we put the electric field in the set of equation equal to zero. If we put charge of particles equal to zero we get quasi-magnetostatic spin waves [26]- [28]. But in our paper we include a contribution of the charges.

Thus, for spin waves the amplitude of the electric field of wave $\mathbf{E}=0$, whereas the magnetic field in the wave is nonzero, $\mathbf{B} \neq 0$. In system of equations (4) and (6), we assume $\mathbf{E}=0$, and obtain the following equation:

$$
\Pi_{\alpha \beta}(\omega, \mathbf{k}) \delta B_{\beta}(\omega, \mathbf{k})=0 .
$$

From equation (22) we have dispersion equation

$$
\operatorname{det} \widehat{\Pi}=0 .
$$

We do not present explicit form of this equation because of its largeness. Explicit form of the matrix $\Pi_{\alpha \beta}(\omega, \mathbf{k})$ is presented in [24]. Here we consider influence of the spin-orbit interaction. This interaction realized by means of electric field. Consequently, there is no contribution of spin-orbit interaction in (22). In fact, we consider the equation obtained earlier [24]. But, we present more detailed analysis of this formula. Propagation of spin waves along the direction of the external magnetic field was considered in [24]. Here we study propagation of the spin waves in the whole $\mathbf{k}$ space.

There are three solutions of dispersion equation $\operatorname{det} \hat{\Pi}=$ 0 for the case of the spin waves propagating parallel to the
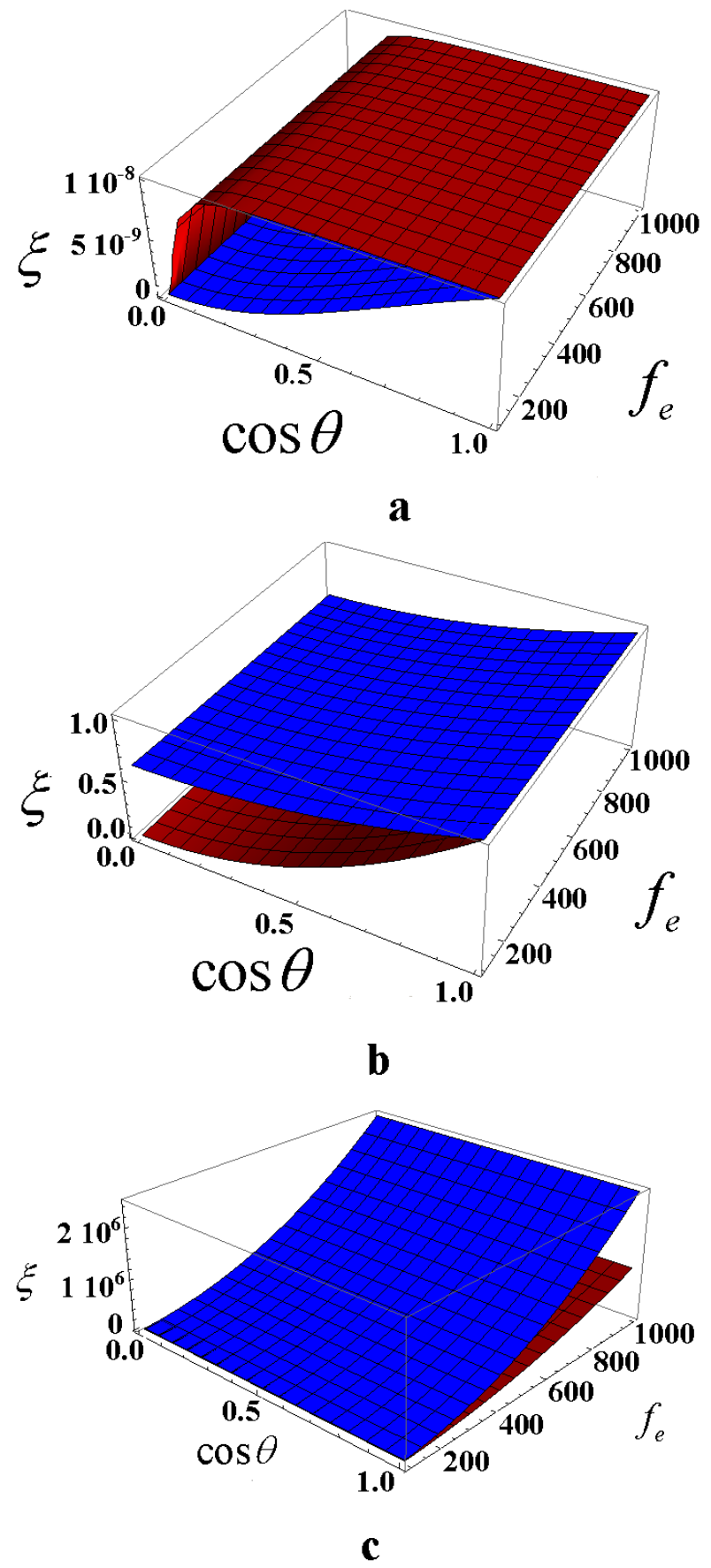

FIG. 1. This figure displays the dispersion dependence determined by equation (28) (in fact it is the function $\omega(k)$ ). Equation (28) has three solutions. We present this solution on fig a, b and c, correspondingly. Each solution is obtained for two different values of $\chi=\chi_{i} / \chi_{e}$. Here we interested in the cases when $\chi_{e}$ and $\chi_{i}$ have the same order or differ by several orders. For room temperature we suppose $f_{i} \simeq 10^{-3} f_{e}$ and approximately take $g \simeq 10^{-4}$. We consider the dense plasma. Therefore, the quantity $f_{e}$ is order of $10^{2}-10^{3}$. Thus, we consider $\xi$ like a function of $k$ (via $\left.f_{e}\right)$ and $\cos \theta: \xi=\xi\left(f_{e}, \cos \theta\right)$. The parameter $\chi$ has a following values. For the first case (blue surface) $\chi=1.5$ and in the second case (red) $\chi=0.002$. 
direction of the external magnetic field. Two solutions are the oscillations with constant frequencies:

$$
\omega=\left|\Omega_{a}\right|\left(1-4 \pi \chi_{a}\right) .
$$

At $m_{e} \chi_{i} \neq m_{i} \chi_{e}$ the solution is

$\omega^{2}=k^{2} \frac{\left(v_{s i}^{2}+\frac{\hbar^{2}}{4 m_{i}^{2}} k^{2}\right) \chi_{e}\left|\Omega_{e}\right|-\left(v_{s e}^{2}+\frac{\hbar^{2}}{4 m_{e}^{2}} k^{2}\right) \chi_{i}\left|\Omega_{i}\right|}{\chi_{e}\left|\Omega_{e}\right|-\chi_{i}\left|\Omega_{i}\right|}$.

This formula represents the dispersion of the selfconsistent spin waves in the system of the electrons and ions with nonzero intrinsic magnetic moments.
Neglecting the quantum Bohm potential (the terms proportional to $\hbar^{2}$ ), from (25) we get

$$
\omega=\tilde{v}_{s p} k,
$$

where

$$
\tilde{v}_{s p}=\frac{v_{s i}^{2} \chi_{e}\left|\Omega_{e}\right|-v_{s e}^{2} \chi_{i}\left|\Omega_{i}\right|}{\chi_{e}\left|\Omega_{e}\right|-\chi_{i}\left|\Omega_{i}\right|}
$$

and $\tilde{v}_{s p}$ do not depend on wave vector $k$.

Let us consider the spin waves in the whole $\mathbf{k}$ space. Further we write the evident form of the dispersion equation (23). We consider the terms proportional to the magnetic susceptibility $\chi_{a}$ in the first and the second degree. The dispersion equation is

$$
\begin{gathered}
\sum_{a} \chi_{a} \frac{\Omega_{a}}{\Delta_{a}}\left(k_{z}^{2}\left(\omega^{2}-\Omega_{a}^{2}\right)+k_{\perp}^{2} \omega^{2}\right) \\
+4 \pi \sum_{a, b} \chi_{a} \chi_{b} \frac{1}{\Delta_{a}} \frac{\Omega_{a} \Omega_{b}}{\omega^{2}-\Omega_{b}^{2}}\left(2 k_{z}^{2} \Omega_{b}\left(\omega^{2}-\Omega_{a}^{2}\right)+k_{\perp}^{2}\left(\Omega_{a}+\Omega_{b}\right) \omega^{2}\right)=0
\end{gathered}
$$

where

$$
\Delta_{a}=\omega^{2}\left(\omega^{2}-\Omega_{a}^{2}\right)-v_{q s a}^{2}\left(\omega^{2} k_{\perp}^{2}+\left(\omega^{2}-\Omega_{a}^{2}\right) k_{z}^{2}\right) .
$$

Equation (26) is the equation of the fifth degree of $\omega^{2}$.

The quantity $\chi_{a}$ is much less than $1: \chi_{a}<<1$. Consequently, in the simplest case, we neglect the terms proportional to $\chi_{a}^{2}$, in comparison with the terms proportional to $\chi_{a}$. We obtain equation of the third degree of $\omega^{2}$.

$$
\sum_{a} \chi_{a} \frac{\Omega_{a}}{\Delta_{a}}\left(k_{z}^{2}\left(\omega^{2}-\Omega_{a}^{2}\right)+k_{\perp}^{2} \omega^{2}\right)=0
$$

or in the explicit form

$$
\begin{gathered}
\left(\chi_{e}+\chi_{i} g\right) \xi^{3} \\
-\left(\left(\chi_{e}+\chi_{i} g^{3}\right) \cos ^{2} \theta+\chi_{e}\left(g^{2}+f_{i}^{2}\right)+\chi_{i} g\left(1+f_{e}^{2}\right)\right) \xi^{2} \\
+\left(\chi_{e}\left(g^{2}+f_{i}^{2}\right) \cos ^{2} \theta+\chi_{i} g^{3}\left(1+f_{e}^{2}\right) \cos ^{2} \theta\right. \\
\left.+g\left(\chi_{e} f_{i}^{2} g+\chi_{i} f_{e}^{2}\right)\right) \xi-g^{2} \cos ^{2} \theta\left(\chi_{e} f_{i}^{2}+\chi_{i} f_{e}^{2} g\right)=0,
\end{gathered}
$$

where $\xi=\omega^{2} / \Omega_{e}^{2}, g=\Omega_{i} / \Omega_{e}, f_{a}^{2}=v_{q s a}^{2} k^{2} / \Omega_{e}^{2}, \theta$ is the polar angle: $\cos \theta=k_{z} / k$ and $k=\sqrt{k_{z}^{2}+k_{\perp}^{2}}$. Equation
$(28)$ is the third degree in $\xi$ and we can not obtain analytical solutions of this equation. Consequently, we present it's numerical solution on Fig. (1). We consider two type of material, the first one with close values of $\chi_{e}$ and $\chi_{i}$ and the second one such that $\chi_{i} \ll \chi_{e}$. is

For one kind of particle there is only one spin mode. It

$$
\omega=\left|\Omega_{a}\right| \cos \theta
$$

Solution (29) is generalization of (24) for whole $\mathbf{k}$ space.

The terms proportional to $\chi_{a}^{2}$ have significant contribution in equation (26) at $\omega^{2}-\Omega_{a}^{2} \approx 0$. In this case we obtain analytical solutions. Subindex $a$ equal $e$ and $i$. Since we have two wave solutions, for each sorts of particles. For both waves the dispersion relation may be presented in the form:

$$
\omega=\left|\Omega_{a}\right|+\delta \omega,
$$

with $a=e, i$. For wave with frequency around the electron cyclotron frequency we have

$$
\delta \omega=\pi \chi_{e} \Omega_{e} \frac{2 \chi_{e} \Omega_{e}\left(\Omega_{e}^{2}-v_{i}^{2} k^{2}\right)-\chi_{i} \Omega_{i} v_{q S e}^{2}\left(2 k_{z}^{2}+k_{\perp}^{2}\right)}{\chi_{e} \Omega_{e}\left(\Omega_{e}^{2}-v_{i}^{2} k^{2}\right)-\chi_{i} \Omega_{i} v_{q S e}^{2} k^{2}},
$$


and around the ion cyclotron frequency

$$
\delta \omega=2 \pi \chi_{i} \Omega_{i} \frac{-2 \chi_{i} \Omega_{i}\left(\left(\Omega_{e}^{2} k_{z}^{2}-\Omega_{i}^{2} k_{\perp}^{2}\right) v_{q S e}^{2}-\Omega_{i}^{2} \Omega_{e}^{2}\right)+\chi_{e} \Omega_{e}^{2}\left(\Omega_{i} k_{\perp}^{2}-2 \Omega_{e} k_{z}^{2}\right) v_{i}^{2}}{\chi_{i} \Omega_{i}\left(\left(\Omega_{e}^{2} k_{z}^{2}-\Omega_{i}^{2} k_{\perp}^{2}\right) v_{q S e}^{2}-\Omega_{i}^{2} \Omega_{e}^{2}\right)+\chi_{e} \Omega_{e}\left(\Omega_{e}^{2} k_{z}^{2}-\Omega_{i}^{2} k_{\perp}^{2}\right) v_{i}^{2}},
$$

where $v_{q s a}^{2}$ defined by the formula (21).

Under the condition $k_{\perp}=0$ solutions (30) and (31) pass to

$$
\delta \omega=2 \pi \chi_{e} \Omega_{e}
$$

for (30), and

$$
\delta \omega=-4 \pi \chi_{i} \Omega_{i},
$$

for 31 .

The spin-orbit interaction has no influence on dynamic of the spin waves in the plasma. We investigate the spin waves like waves in propagation of which the electric field take no part. But force of the spin-orbit interaction is proportional to the electric field. Problem of the generation of the spin-waves which propagate parallel to the direction of the external magnetic field was analyzed in [23].

In this paper we interested in effects existed at propagation of the neutron beam through the plasma parallel to the external magnetic field. In this case there are instabilities of the spin waves, but waves propagated parallel to the external magnetic field, only. Three waves exist at described conditions. Dispersion of this waves presented by formulas (24) and (25). For the last one there is no instability. For the two solutions (24) we obtain following increments of instabilities.

In the absence of the medium, for the spin waves in the neutron beam we get the dispersion relation.

$$
\omega=k U_{z} \pm \Omega_{b}\left(1-4 \pi \chi_{b}\right) .
$$

Under the conditions of resonance $k U_{z}+\varepsilon \Omega_{b}=$ $\left|\Omega_{c}\right|\left(1-4 \pi \chi_{c}\right)$, here $\varepsilon= \pm 1$, the dependence of frequency from wave vector is

$$
\omega=\left|\Omega_{c}\right|\left(1-4 \pi \chi_{c}\right)+\delta \omega .
$$

Using substitution (33), we receive $\delta \omega$ from the dispersion equation. First of them is

$$
\delta \omega^{2}=-(4 \pi)^{2} \chi_{b} \chi_{c}\left|\Omega_{b}\right| \Omega_{c} .
$$

This formula is valid for the electrons at condition $\varepsilon=-1$, and for the ions at $\varepsilon=+1$. Second solution has form

$$
\delta \omega^{2}=-4 \frac{\chi_{b}}{\chi_{c}}\left|\Omega_{b}\right| \Omega_{c},
$$

and valid for the ions at $\varepsilon=-1$ and for the electrons at $\varepsilon=+1$. From formulas (34), (35) we see the solution for the ions wave take place just in case paramagnetic ions.

It is the same results we obtained in paper [23], because the spin-orbit interaction has no influence on the spin waves and their instabilities. Instabilities of the spin waves there are due to the spin-spin and the spin-current interactions. This interaction holds between magnetic moments of the beam and electric currents and magnetic moments of the plasma.

\section{CONCLUSION}

In this work we consider the influence of the magnetic moment or spins of particles on dynamics of the magnetized plasma.

We discovered two new waves propagating in the plasma along the external magnetic field. We obtained the four new wave propagating in the plasma perpendicular to the direction of the magnetic field. We studied possibility of existence of the spin waves and obtained the dispersion of the spin waves.

We show existence of resonant interaction of the neutron beam with the plasma. This effect leads to instabilities in the plasma. Consequently, there is generation of the waves in the plasma by means of the neutron beam.

For generation of waves arisen from dynamic of magnetic moments the neutron beam is more useful in compare with the beam of electrons. It is because the beam of electrons, first of all, excites the waves of charge.

\section{APPENDIX}

In section II, we present the Schrodinger equation contained only the Coulomb interaction. In this paper we interested in the spin-spin, the spin-current and the spin-orbit interactions. The whole Hamiltonian, used in this work reads

$$
\hat{H}=\sum_{p}\left(\frac{1}{2 m_{p}} \tilde{D}_{p}^{\alpha} \tilde{D}_{p}^{\alpha}+e_{p} \varphi_{p}^{e x t}-\gamma_{p} \sigma_{p}^{\alpha} B_{p(e x t)}^{\alpha}\right)-\sum_{p} \frac{\gamma_{p}}{m_{p} c} \varepsilon^{\alpha \beta \gamma} \sigma_{p}^{\alpha} E_{p, e x t}^{\beta} \hat{D}_{p}^{\gamma}
$$




$$
+\frac{1}{2} \sum_{p, n \neq p}\left(e_{p} e_{n} G_{p n}-\gamma_{p} \gamma_{n} G_{p n}^{\alpha \beta} \sigma_{p}^{\alpha} \sigma_{n}^{\beta}\right)+\sum_{p, n \neq p} \frac{e_{p}}{m_{n} c} \lambda \gamma_{n} \varepsilon^{\alpha \beta \gamma} \partial_{n}^{\gamma} G_{p n} \sigma_{n}^{\beta} \hat{D}_{n}^{\alpha}
$$

where

$$
\begin{gathered}
D_{p}^{\alpha}=-\imath \hbar \partial_{p}^{\alpha}+\frac{e_{p}}{c} A_{p, e x t}^{\alpha}, \\
\left(\tilde{D}_{p}^{\alpha} \psi\right)_{s}(R, t)=\left(\left(\frac{\hbar}{\imath} \partial_{p}^{\alpha}-\frac{e_{p}}{c} A_{p(e x t)}^{\alpha}-\frac{e_{p}}{c} \sum_{n \neq p}\left(\frac{\xi}{2}\right) \varepsilon^{\alpha \beta \gamma} \frac{r_{n p}^{\beta}}{r_{n p}^{3}} \gamma_{n} \sigma_{n}^{\gamma}\right) \psi\right)_{s}(R, t),
\end{gathered}
$$

here $\xi$ is an arbitrary numerical parameter. We choose the value of $\xi$ so that the superposition principle of fields holds. We consider superposition of the external magnetic field and the field caused by the magnetic moments and the electric currents. From calculation we get $\xi=2$.

Here, we describe the meaning of terms in the Hamiltonian (36). The first term has a complex structure. This is the kinetic energy of the particles, where we include vector potential of magnetic field caused by spins, along with the external field. Thereby, the first term include the spincurrent interaction. The second and third terms are the action of the external field on the charges and the magnetic moments. Next term is effect of the external electric field on moving magnetic moments or the spin-orbit interaction with the external electric field [29]. The first and second terms in the second line are the Coulomb and the spin-spin interaction. And, the last one is the spin-orbit interaction between particles.

The Green's functions of the Coulomb, the spin-spin and the spin-current interactions has the following form $G_{p n}=1 / r_{p n}, G_{p n}^{\alpha \beta}=4 \pi \delta^{\alpha \beta} \delta\left(\mathbf{r}_{p n}\right)+\partial_{p}^{\alpha} \partial_{p}^{\beta}\left(1 / r_{p n}\right)$, $C_{p n}^{\alpha \beta}=\left(e_{n} / c\right) \varepsilon^{\alpha \beta \gamma} r_{p n}^{\gamma} / r_{p n}^{3}$, where $\gamma_{p}$ - is the gyromagnetic ratio. For electrons $\gamma_{p}$ reads $\gamma_{p}=e_{p} \hbar /\left(2 m_{p} c\right), e_{p}=$ $-|e|$. The quantities $\varphi_{p}^{e x t}=\varphi\left(\mathbf{r}_{p}, t\right), A_{p(e x t)}^{\alpha}=A^{\alpha}\left(\mathbf{r}_{p}, t\right)$ are the scalar and the vector potentials of the external electromagnetic field:

$$
\begin{gathered}
B_{(e x t)}^{\alpha}\left(\mathbf{r}_{p}, t\right)=\varepsilon^{\alpha \beta \gamma} \nabla_{p}^{\beta} A_{(e x t)}^{\gamma}\left(\mathbf{r}_{p}, t\right), \\
E_{(e x t)}^{\alpha}\left(\mathbf{r}_{p}, t\right)=-\nabla_{p}^{\alpha} \varphi_{e x t}\left(\mathbf{r}_{p}, t\right)-\frac{1}{c} \frac{\partial}{\partial t} A_{e x t}^{\alpha}\left(\mathbf{r}_{p}, t\right) .
\end{gathered}
$$

$\sigma_{p}^{\alpha}$ is the Pauli matrix, a commutation relations for them is

$$
\left[\sigma_{p}^{\alpha}, \sigma_{n}^{\beta}\right]=2 \imath \delta_{p n} \varepsilon^{\alpha \beta \gamma} \sigma_{p}^{\gamma} .
$$

The Hamiltonian (36) is an analog of the Breit's Hamiltonian [29] and [30]. Here we do not consider the semirelativistic contribution in the kinetic energy $\sim \mathbf{D}_{p}^{4}$ and the current-current interaction (the Biot-Savart law). Nonquantum part of the last one we include by means of superposition principle of fields.

Using the Hamiltonian (36) we obtained the QHD equation contained many-particle correlations. This correlations include the exchange interaction. We make notice here about the exchange interaction existing for all kind of forces between particles. They are the Coulomb, the spinspin, the spin-current and the spin-orbit interactions. The equations of the momentum balance and magnetic moment evolution arise in the form of integro-differential equations. This form useful for studying low dimensional systems (see for example [14]). In this work we neglected correlations and use the self-consistent field approximation. We made also the additional approximations. We account terms coincided to the following non-linear one-particle Schrodinger equation

$$
\begin{gathered}
\imath \hbar \partial_{t} \Phi(\mathbf{r}, t)=\frac{1}{2 m} \widehat{\mathbf{D}}^{2} \Phi(\mathbf{r}, t)-\mu \widehat{\sigma}^{\alpha} B^{\alpha}(\mathbf{r}, t) \Phi(\mathbf{r}, t) \\
+e \varphi(\mathbf{r}, t) \Phi(\mathbf{r}, t)-\frac{\gamma}{m c} \varepsilon^{\alpha \beta \gamma} \widehat{\sigma}^{\alpha} E^{\beta}(\mathbf{r}, t) \widehat{D}^{\gamma}(\mathbf{r}, t) \Phi(\mathbf{r}, t),
\end{gathered}
$$

where

$$
\widehat{\mathbf{D}}=\widehat{\mathbf{D}}(\mathbf{r}, t)=\widehat{\mathbf{p}}-(e / c) \mathbf{A}(\mathbf{r}, t)
$$

and

$$
\Phi^{*}(\mathbf{r}, t) \Phi(\mathbf{r}, t)=n(\mathbf{r}, t) .
$$

Here we would like to justify the choice of form of the Hamiltonian (36), especially of the first term contained the spin-current interaction. For this purpose we consider classic Lagrangian. For one charged particle particle in the external electromagnetic field the Lagrangian has form:

$$
L=\frac{m \mathbf{v}^{2}}{2}-e \varphi+\frac{e}{c} \mathbf{v A} .
$$

For particles having magnetic moment there is additional term to the potential energy, this term has form

$$
\Delta U=-\mu^{\alpha} B^{\alpha} \text {. }
$$

In this case the Lagrangian is

$$
L=\frac{m \mathbf{v}^{2}}{2}-e \varphi+\frac{e}{c} \mathbf{A} \mathbf{v}+\mu^{\alpha} B^{\alpha} .
$$

Starting from this point we can build Lagrangian for $N$ interacting particles

$$
L=\sum_{i} \frac{m_{i} \mathbf{v}_{i}^{2}}{2}-\sum_{i, j \neq i} e_{i} e_{j} G_{i j}
$$




$$
+\sum_{i}\left(\frac{e_{i}}{c} \mathbf{v}_{i} \mathbf{A}_{i}+\mu_{i}^{\alpha} B_{i}^{\alpha}\right)
$$

where $\mathbf{A}_{i}, \mathbf{B}_{i}$ describe magnetic fields made by $j$-th particles and act on $i$-th particle. We do not consider the external field for compactness. The sources of the magnetic field are the moving charges

$$
\mathbf{A}_{i}=\sum_{j \neq i} \frac{e_{j}}{c} \frac{\mathbf{v}_{j}}{r_{i j}}
$$

and the magnetic moments

$$
A_{i}^{\alpha}=-\sum_{j \neq i} \varepsilon^{\alpha \beta \gamma} \frac{r_{i j}^{\alpha}}{r_{i j}^{3}} \mu_{j}^{\gamma} .
$$

We do non considered the current-current interaction, which is proportional to the square of the velocity. Magnetic field has form

$$
B_{i}^{\alpha}=\sum_{j \neq i} G_{i j}^{\alpha \beta} \mu_{j}^{\beta}
$$

caused by magnetic moments and

$$
B_{i}^{\alpha}=-\sum_{j \neq i} \varepsilon^{\alpha \beta \gamma} \frac{e_{j}}{c} \frac{r_{i j}^{\beta}}{r_{i j}^{3}} v_{j}^{\gamma}
$$

caused by moving chargees. The quantity $G_{i j}^{\alpha \beta}$ is the Green's function of the spin-orbit interaction presented above. In this way the Lagrangian (38) is

$$
\begin{gathered}
L=\sum_{i}\left(\frac{m_{i} \mathbf{v}_{i}^{2}}{2}-e_{i} \varphi_{i}^{e x t}+\frac{e_{i}}{c} \mathbf{v}_{i} \mathbf{A}_{i}^{e x t}+\mu_{i}^{\alpha} B_{i}^{\alpha e x t}\right) \\
+\sum_{i, j \neq i}\left(-\frac{1}{2} e_{i} e_{j} G_{i j}+\frac{1}{2} G_{i j}^{\alpha \beta} \mu_{i}^{\alpha} \mu_{j}^{\beta}+\frac{e_{i}}{c} \varepsilon^{\alpha \beta \gamma} \mu_{j}^{\alpha} r_{i j}^{\beta} v_{i}^{\gamma} \frac{1}{r_{i j}^{3}}\right),
\end{gathered}
$$

here we include external field. The corresponding Hamiltonian is

$$
\begin{gathered}
H=\sum_{i} \frac{1}{2 m_{i}}\left(\mathbf{p}_{i}-\frac{e_{i}}{c} \mathbf{A}_{i}^{e x t}-\frac{e_{i}}{c} \sum_{j \neq i} \varepsilon^{\alpha \beta \gamma} \mu_{j}^{\beta} r_{i j}^{\gamma} \frac{1}{r_{i j}^{3}}\right)^{2} \\
+\sum_{i}\left(e_{i} \varphi_{i}^{e x t}-\mu_{i}^{\alpha} B_{i}^{\alpha e x t}\right)+\frac{1}{2} \sum_{i, j \neq i}\left(e_{i} e_{j} G_{i j}-\mu_{i}^{\alpha} \mu_{j}^{\beta} G_{i j}^{\alpha \beta}\right) .
\end{gathered}
$$

Using the usual quantization method for the Hamiltonian (39) we obtain (36), except the spin-orbit interaction.

\footnotetext{
* andreevpa@physics.msu.ru

† lsk@phys.msu.ru
}

[1] R. B. Miller, An Introduction to the Physics of Intense Charged Particle Beams (Plenum, New York, 1982).

[2] S. Ichimaru, Basic Principles of Plasma Physics (W. A. Benjamin, Inc., Reading, Massachusetts, 1973).

[3] A. B. Mikhailovskii, Theory of Plasma Instabilities (Consultant Bureau, New York, 1974).

[4] A. Bret, L. Gremillet, D. Benisti, and E. Lefebvre, Phys. Rev. Lett. 100, 205008 (2008).

[5] A. Bret, M.-C. Firpo, and C. Deutsch, Phys. Rev. E 70, 046401 (2004).

[6] L. Gremillet, D. Benisti, E. Lefebvre, A. Bret, Physics of plasmas 14, 040704 (2007).

[7] E. Nakar, A. Bret, and M. Milosavljevic, The Astrophysical Journal, 738, 93 (2011).

[8] A. Bret, C. Deutsch, Physics of plasmas 13, 042106 (2006).

[9] I. V. Timofeev, K. V. Lotov, and A. V. Terekhov, Physics of plasmas 16, 063101 (2009).

[10] A. Bret and F. Haas, Physics of plasmas 18, 072108 (2011).

[11] A. Bret and M. E. Dieckmann, Physics of plasmas 15, 062102 (2008).

[12] P. K. Shukla, B. Eliasson, Phys. Usp. 5351 (2010) [Uspehi Fizihceskih Nauk 180, 55 (2010)].

[13] P. K. Shukla, B. Eliasson, Rev. Mod. Phys. 83, 885 (2011).

[14] P. A. Andreev, L. S. Kuz'menkov, M. I. Trukhanova, Phys. Rev. B 84, 245401 (2008).

[15] F. A. Asenjo, J. Zamanian, M. Marklund, G. Brodin, and P. Johansson, arXiv:1108.4781.

[16] L. S. Kuz'menkov and S. G. Maksimov, Teor. i Mat. Fiz., 131231 (2002) [Theoretical and Mathematical Physics 131 641 (2002)].

[17] Yu. Tyshetskiy, S.V. Vladimirov, R. Kompaneets, arXiv:1108.0988.

[18] L. S. Kuz'menkov and S. G. Maksimov, Teor. i Mat. Fiz., 118287 (1999) [Theoretical and Mathematical Physics 118 227 (1999)].

[19] L. S. Kuz'menkov, S. G. Maksimov, and V. V. Fedoseev, Theor. Math. Fiz. 126136 (2001) [Theoretical and Mathematical Physics, 126110 (2001)].

[20] M. Marklund and G. Brodin, Phys. Rev. Lett. 98, 025001 (2007).

[21] F. A. Asenjo, V. Muoz, J. A. Valdivia, and S. M. Mahajan, Phys. Plasmas 18, 012107 (2011).

[22] P. A. Andreev, L. S. Kuz'menkov, Phys. Rev. A 78, 053624 (2008).

[23] P. A. Andreev, L.S. Kuz'menkov, Physics of Atomic Nuclei 71, N.10, 1724 (2008).

[24] P. A. Andreev, L.S. Kuz'menkov, Moscow University Physics Bulletin 62, N.5, 271 (2007).

[25] P. A. Andreev and L. S. Kuz'menkov, PIERS Proceedings, pp.1047, March 20-23, Marrakesh, MOROCCO 2011.

[26] G. A. Maugin, A. Fomethe, International Journal of Engineering Science 20, 885 (1982).

[27] A. A. Barybin, The European Physical Journal Applied Physics 22, 189 (2003).

[28] A. Unal Erdem, Vibration Problems ICOVP 2005, Springer Proceedings in Physics 111, 175 (2007).

[29] V.B. Berestetskii, E.M. Lifshitz, L.P. Pitaevskii, Quantum Electrodynamics, Vol. 4, 2nd ed. (Butterworth-Heinemann, 1982).

[30] G. Breit, Phys. Rev. 34, 553 (1929); V. Yu. Lazur, S. I. Myhalyna, and O. K. Reity, Phys. Rev. A 81, 062707 (2010). 